ASTHMA

\title{
Doubling the dose of budesonide versus maintenance treatment in asthma exacerbations
}

\author{
J M FitzGerald, A Becker, M R Sears, S Mink, K Chung, J Lee, and the Canadian Asthma \\ Exacerbation Study Group
}

Thorax 2004,59:550-556. doi: 10.1136/thx.2003.014936

\begin{abstract}
See end of article for authors' affiliations .....................

Correspondence to: Dr J M FitzGerald, Centre for Clinical Epidemiology and Evaluation, VGH Research Pavilion, 822 West 10th Avenue, Vancouver, BC, V5Z 1 L8, Canada; markf@ interchange.ubc.ca
\end{abstract}

Received 19 August 2003 Accepted 7 February 2004

\begin{abstract}
Background: Previous guidelines recommend doubling the daily dose of maintenance inhaled corticosteroid to treat or prevent progression of exacerbations of asthma.

Methods: Over a 6 month period a cohort of patients were evaluated prospectively and randomised in a double blind controlled trial to treatment with either a continued maintenance dose (MD) of inhaled corticosteroid or doubling the dose (DD) at the time of an exacerbation.

Results: A total of 290 patients were randomised ( $33 \%$ male) and 98 (DD, $n=46$ ) experienced evaluable asthma exacerbations during the study period. Mean (SD) baseline characteristics at randomisation (age 33.5 (14.0) years; forced expiratory volume in 1 second $\left(\mathrm{FEV}_{1}\right) 2.8$ (0.7) I; peak expiratory flow (PEF) 422.9 (110.5) I/min) were similar in both groups. In the DD group $41 \%$ of patients were considered treatment failures because they either required systemic steroids $(n=12)$, had an unscheduled visit to a physician $(n=1)$, or their asthma did not return to baseline $(n=6)$. This did not differ from the MD group in which $40 \%$ were treatment failures $(n=9,0$, and 12, respectively; $p=0.94$ ).
\end{abstract}

Conclusions: In patients who regularly take an inhaled corticosteroid, doubling the maintenance dose may not affect the pattern of the exacerbation.
A sthma exacerbations are common and, in recent years, there has been an increase in the prevalence of asthma and asthma related morbidity. ${ }^{1}$ The management of asthma currently focuses on the regular use of antiinflammatory treatment primarily with an inhaled corticosteroid (ICS). ${ }^{2}$ Previous asthma guidelines have recommended the use of an action plan to manage asthma exacerbations. ${ }^{3}$ Many of these plans advocate a doubling of the dose of maintenance ICS as one of the first steps in the management of worsening asthma. ${ }^{4}$ More recent guidelines have been more cautious and recognise the absence of evidence for this recommendation. ${ }^{5}{ }^{6}$ Lahdensuo $e t ~ a l^{7}$ investigated the effect of supervised patient self-management of exacerbations of asthma, including doubling the dose of ICS for 2 weeks if required, compared with conventional treatment and found statistically significant reductions in unscheduled outpatient visits, days off work, courses of prednisolone and of antibiotics, and improvements in quality of life in the selfmanagement group. However, the trial did not directly examine the effect of doubling the dose of ICS on worsening of exacerbations.

In 1995, while developing the first national evidence based asthma guidelines, we found no randomised controlled trials to support doubling the dose of ICS on identification of exacerbations of asthma. ${ }^{3}$ Asthma exacerbations are common, even in mild asthma, ${ }^{8}$ and therefore an appropriate strategy for their management is required. A number of different end points might be used to evaluate the response to an increase in the dose of ICS, but we were primarily interested in progression to a more severe exacerbation. We therefore addressed the question of whether doubling the dose of maintenance inhaled budesonide early in an asthma exacerbation prevents worsening and the need for systemic corticosteroid. Some of these results have been previously reported in the form of an abstract. ${ }^{9}$

\section{METHODS}

\section{Patients and study design}

Following a 3-6 week run in period, a 6 month double blind, randomised, placebo controlled, parallel group, multicentre trial was carried out in university affiliated teaching hospitals between 1998 and 1999. During the run in period patients using pressurised metered dose inhalers and other forms of inhalers (such as Diskhaler) were converted to budesonide (Pulmicort, Turbuhaler) and monitored to demonstrate asthma stability.

The following were the main inclusion criteria for the study: age $\geqslant 13$ years, documentation of the diagnosis of asthma within the previous year (up to and including the first study visit) as shown by at least one of the following: $\geqslant 12 \%$ reversibility in forced expiratory volume in 1 second $\left(\mathrm{FEV}_{1}\right)$ post bronchodilator, concentration of methacholine provoking a fall in $\mathrm{FEV}_{1}$ of $\geqslant 20 \%\left(\mathrm{PC}_{20}\right)<8 \mathrm{mg} / \mathrm{ml}$ (with an $\mathrm{FEV}_{1}>80 \%$ of predicted normal when no bronchodilators were used within the previous 6 hours), or $\geqslant 20 \%$ diurnal variability in PEF as defined by ( (best PEF - worst PEF)/best $\mathrm{PEF}) \times 100$.

Patients were also required to be on a stable dose of ICS $(\leqslant 1200 \mu \mathrm{g} /$ day beclomethasone or equivalent twice daily regimen) for 1 month before visit 1 and to have had at least one previous asthma exacerbation, defined as an increase in symptoms necessitating a change in medication, not more than 12 months and not less than 1 month before the start of the run in period. In addition, patients were required to have adequate skill or the potential to learn the proper use of the Turbuhaler inhaler, the computerised Vitalograph, and the MiniDoc.

Patients who had had an exacerbation (as defined in the inclusion criteria) in the month before visit 1 , patients whose exacerbations were due to chronic sinusitis as judged by the investigator, those with a history of near fatal asthma 
(requiring intubation and mechanical ventilation) in the preceding 10 years, hospitalisation due to asthma in the 3 months before visit 1 , and patients who regularly used oral or parenteral glucocorticosteroids during the month before visit 1 were excluded from the study. Treatment with ketotifen, disodium cromoglycate and/or nedocromil sodium during the month before visit 1 , treatment with long acting $\beta_{2}$ agonists, and respiratory tract infection within 1 month of visit 1 were further reasons for exclusion. Current smokers and patients with a smoking history of 10 pack years or more, pregnant or lactating women, or women of child bearing potential not using an effective means of birth control were also excluded.

The study protocol was approved by the institutional ethics committees at each research site and signed written informed consent was received from all patients prior to enrolment.

\section{Study procedures}

After the run in period the patients were randomised to one of two treatment arms. The maintenance dose (MD) group received a maintenance inhaler of budenoside dispensing 100,200 , or $400 \mu \mathrm{g} /$ dose (depending on their maintenance therapy) plus an additional inhaler containing placebo for twice daily use. The double dose (DD) group received the same maintenance inhaler as the first group, but the additional inhaler dispensed 100, 200, or $400 \mu \mathrm{g} / \mathrm{dose}$ of budesonide as well. The additional Turbuhaler was identical in appearance to the maintenance Turbuhaler but was identifiable by a red dot. In both treatment arms the additional inhaler was to be added to the twice daily maintenance inhaler at the time of an asthma exacerbation. The time of the exacerbation was deemed to have begun when the criteria outlined below were present for 48 hours. Thus, upon exacerbation, one group received a doubling dose of ICS while the dose received by patients in the other group remained unchanged.

Patients not currently using budesonide were switched to it at an equivalent dose and placed on a twice daily dosage regimen. The dosage conversion was as close to a $1: 1$ ratio as possible, based on the investigator's clinical judgement. All patients received a terbutaline sulphate inhaler (Bricanyl Turbuhaler, $500 \mu \mathrm{g} /$ inhalation as required) to be used as rescue medication throughout the study. Each patient received a 7 day supply of oral methylprednisolone (MP) totalling $32 \mathrm{mg} /$ day to be used, if needed, to treat an exacerbation.

$\mathrm{FEV}_{1}$ was measured in all patients during the initial clinic visit. PEF data were obtained using the Vitalograph 2110 computerised peak flow meter and recorded in an electronic diary (MiniDoc). These measurements were collected morning and evening during the run in and throughout the study period. The morning value was used to assess for an exacerbation. Diary data were also to be used as criteria in the definition of an exacerbation during the treatment period. Symptoms from the previous 24 hours were used to create a score. The symptoms included chest tightness, breathlessness, coughing, and wheezing. The symptoms were rated each morning according to the following scale: $0=$ none, $1=$ mild, $2=$ moderate, and $3=$ severe. The alert PEF value was $80 \%$ of the mean baseline morning PEF. The Vitalograph displayed different colour zones corresponding to percentage ranges of the patient's baseline PEF value: the green zone represented $81-100 \%$, the yellow represented 61$80 \%$, and the red zone $\leqslant 60 \%$ of the baseline morning PEF. The mean baseline PEF was calculated from the 7 days immediately preceding visit 2 .

When a patient performed a PEF test the Vitalograph recorded and displayed the numerical value and indicated by means of an arrowhead the colour zone in which the attempt fell. Patients then entered the colour zone results into the MiniDoc. The alert asthma symptom score (three ordinal values above the mean baseline total symptom score on two consecutive days) was calculated on an individual patient basis and programmed into the MiniDoc. The MiniDoc alerted the patient in the event of an asthma exacerbation. Once the MiniDoc indicated to a patient that the criteria for an exacerbation had been fulfilled, the patient reported this to the study nurse or physician and was given instructions to begin taking the additional inhaler. A critical PEF value of $60 \%$ of the mean baseline morning PEF-that is, a $40 \%$ fall in the mean baseline morning $\mathrm{PEF}$-was also programmed into the MiniDoc. If this lower level was reached, the MiniDoc signalled the patient to begin oral MP. The critical PEF value was derived from patient groups involved in previous action plans used in education intervention studies. ${ }^{7}$

\section{Outcomes}

The primary outcome variable was defined as the proportion of patients who, after developing an exacerbation of their asthma, failed to regain control after introducing the additional inhaler, as judged by the need for treatment with oral MP or an unscheduled visit to a physician or medical emergency department due to asthma or unstable asthma after 14 days of treatment. The question regarding any unscheduled visit was asked at day 14 after the exacerbation.

For the purpose of this study, stability was defined as a morning PEF of $\geqslant 90 \%$ of the mean baseline value on either of the previous two days, Bricanyl Turbuhaler $<4$ inhalations/ day over the previous two days, no nocturnal awakenings due to asthma over the previous two consecutive nights, and a total symptom score not exceeding the mean baseline level by more than two ordinal values over the previous two days. If these criteria were not met the asthma was deemed to be unstable.

During the study period monthly check up visits were scheduled to ensure that no asthma exacerbations were missed and to encourage patient compliance.

\section{Compliance}

At visit 1 the importance of complying with the dosage regimen was emphasised. Patients were instructed to enter the number of doses taken from their maintenance and additional Turbuhaler into the MiniDoc computerised diary. At each subsequent visit and during any telephone contact, the need for compliance was further reinforced. At all visits subsequent to visit 1 , compliance with the maintenance Turbuhaler was checked by collection of self-reported datathat is, the number of doses of ICS entered in the MiniDoc during the treatment period immediately preceding the visit.

\section{Randomisation procedure}

Patients were randomised to treatment groups at visit 2 according to a blocked computer generated randomisation list for each centre.

\section{Other treatment}

Theophylline, anticholinergics, and nasal steroids were allowed throughout the study.

\section{Asthma exacerbation during treatment period}

An exacerbation during the treatment period-that is, an indication to introduce the additional inhaler-was defined as a combination of two of the following criteria, provided that at least one of the two was the first, second, or third criterion: 
- best PEF test falls to $\leqslant 80 \%$ of the mean baseline morning value on two consecutive readings or two consecutive morning readings;

- bronchodilator use $\geqslant 4$ inhalations/day on two consecutive days;

- nocturnal awakenings due to asthma on two consecutive nights;

- total asthma symptom score for the combined symptoms of chest tightness, breathlessness, coughing, and wheezing increases by $\geqslant 3$ ordinal values from the mean baseline value on two consecutive days;

- inability to go to school or work due to asthma for two consecutive days; and

- unscheduled physician visit due to asthma during the time period when the concomitant criterion/criteria started to be fulfilled.

\section{Three month surveillance period}

Patients who had an asthma exacerbation during the study period and who were assessed as stable at the end of the 14 day additional treatment course were followed for a 3 month surveillance period to monitor their asthma control. This surveillance period was primarily to ensure that there were no late differences shown between the groups.

\section{Data analysis}

The primary objective of this study was to determine whether doubling the dose of budesonide early in the course of an asthma exacerbation is an appropriate strategy for preventing worsening of the exacerbation. The primary efficacy variable was treatment failure-that is, the proportion of patients who, after developing an exacerbation, needed at least one course of oral MP or an unscheduled visit to a physician or medical emergency department due to asthma or who had unstable asthma after 14 days of treatment. It was assumed that the rates of exacerbation would be the same in the two treatment groups. For morning PEF and total asthma symptom score, a mean baseline value was calculated for each patient. The data from the 7 days immediately preceding randomisation (visit 2) were used to make this calculation.

Statistical analysis used the "all patients treated" (APT) approach. Since patients were "treated" only if they had an exacerbation, all patients who had at least one asthma exacerbation after randomisation and were treated with at least one dose of additional study drug are included. The reason for adjusting for the baseline dose of ICS, which was done by post-stratification, was to see the impact of asthma severity on outcomes. The primary variable was analysed using a logistic regression model with adjustments for centre and strata $(\leqslant 400 \mu \mathrm{g}$ ICS $v>400 \mu \mathrm{g}$ ICS $)$ effects. The Cochran-Mantel-Haenszel (CMH) test adjusted for centre was used for analysis of the secondary variable, which was the number of asthma exacerbations during the 3 month surveillance period after the initial exacerbation. SAS version 6.12 running under Windows NT was used for analysis.

\section{Sample size determination}

The sample size was based on two assumptions. Firstly, based on previous studies, it was assumed that $25 \%$ of patients in each treatment group would suffer an exacerbation after randomisation. ${ }^{10}$ Secondly, it was assumed that the proportion of patients suffering an exacerbation who would need a course of oral steroids would be $50 \%$ in the MD group and $20 \%$ in the DD group.

Based on these figures, it was estimated that 38 exacerbations would be needed in each group for a statistical analysis with a significance level of 5\% (two sided) and power of $80 \%$ to show a difference between the two treatment groups. Since the number of exacerbations was a random variable in each group and because imbalance causes a decrease in power, a 5\% increase in the estimated number of exacerbations was considered necessary. This led to an estimated requirement of 160 patients randomised into each treatment group. The 5\% increase was deemed sufficient to compensate for an imbalance of exacerbations in the two groups of $40 / 60 \%$.

\section{Interim analysis}

A review of the exacerbation rate without breaking the treatment code was planned when data for approximately $25 \%$ of the patients were available. If exacerbations were found to be occurring at a sufficient rate, the study was to continue. If not, the protocol was to be amended to increase the sample size and/or lengthen the enrolment period. At the time of the interim analysis it was deemed that an adequate number of exacerbations were occurring.

\section{RESULTS}

The scheme for patient recruitment and randomisation is shown in fig 1 . Sixteen patients discontinued other than for exacerbations (nine from the placebo group). There were no differences in patient characteristics between those who experienced an exacerbation and those who did not (data not shown). Characteristics at randomisation of patients who had an exacerbation of their asthma are shown in table 1. Two of the 52 patients in the MD group were on theophylline and 13 were on constant doses of nasal steroids, while 12 of the 46 patients in the DD group were on similar stable doses of nasal steroids. No patients were taking long acting $\beta$ agonists during the study period.

Table 2 shows the results for the primary outcome variable (treatment failure). No difference was found between treatments $(p=0.94)$. Furthermore, of those patients who completed the 3 month surveillance period following their asthma exacerbation, there was no difference between groups in the mean number of exacerbations (six of 35 in the MD group $v$ five of 34 in the DD group, $p=0.92)$. The median time from the use of additional Turbuhaler-that is, the time from asthma exacerbation to the start of oral MP treatment-was 3 days in both the MD and DD groups.

The pattern of $\beta_{2}$ agonist use, mean symptom scores, and nocturnal awakenings were similar in both arms of the study at the time that patients had an exacerbation (fig $2 \mathrm{~A}-\mathrm{C}$ ). There were no significant differences in the three major criteria for triggering an asthma exacerbation (changes in PEF, increased bronchodilator use, nocturnal awakenings). Because of a technical fault in the recovery of the raw PEF data at the end of the study, the PEF data were flawed and too inconsistent to be analysed at the data centre. These data were, however, available to individual patients to enter into their Mini-Docs throughout the study.

There was no difference in outcome between patients who were switched from other ICS to budesonide $(n=40)$ compared with patients who were already taking budesonide $(n=58)$ when recruited into the study. There were also no differences between centres. Patients receiving ICS in a dose of $\leqslant 400 \mu \mathrm{g} /$ day were less likely to have treatment failure after exacerbation than those receiving ICS in a dose of $>400 \mu \mathrm{g} /$ day in both treatment groups (table 3 ). There was no difference in outcome related to age (adolescents versus adults). The difference with 95\% confidence intervals between treatments in proportions (DD - MD) was $0.9 \%$ (95\% CI -17.9 to 19.8 ).

Compliance with inhaled treatment before an exacerbation, based only on reported use, was high in both groups 


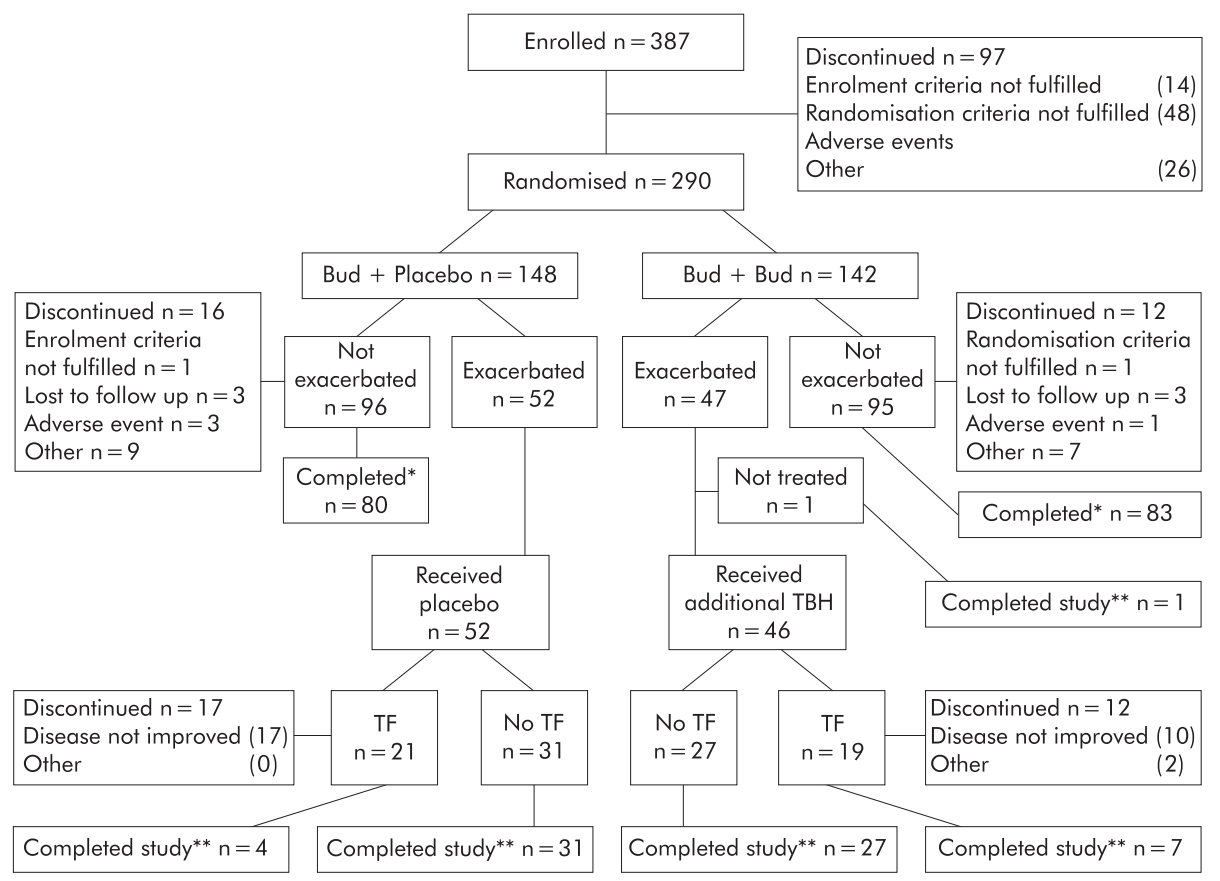

Figure 1 Scheme showing recruitment and subsequent outcome of patients recruited into the study. ${ }^{*}$ Completed visit 8 . ${ }^{*}$ Completed 3 month surveillance period. Bud = budesonide; $\mathrm{TBH}=$ Turbuhaler; $\mathrm{TF}=$ treatment failure.

(mean (SD) 97.2 (11.3)\% in the MD group who did not have an exacerbation and $98.3(4.4) \%$ in the MD group who did compared with 99 (11.2)\% in the DD group who did not have an exacerbation and 96.5 (15.1)\% in the DD group who did). Compliance was calculated based on the best case scenariothat is, replacing missing data with the mean values. Compliance of patients using maintenance ICS during the 14 days after an exacerbation was also excellent (103.3 $(12.2) \%$ in the MD group and $100.7(16.0) \%$ in the DD group). Using the worst case scenario-that is, considering missing values as "medication not taken" and replacing with zero-compliance after an exacerbation was $85.9(22.7) \%$ and $81.5(25.9) \%$ for the MD and DD groups, respectively.

\section{DISCUSSION}

It is common practice to recommend doubling the dose of ICS at the onset of an exacerbation of asthma. Many national and international guidelines for asthma management have previously strongly endorsed this recommendation, but recent guidelines have been more cautious. ${ }^{5}{ }^{6}$ Our study has shown that, in patients using regular ICS, doubling the dose within 48 hours of the onset of an exacerbation did not change the outcome and the need for further intervention compared with patients who continued on their usual maintenance dose.

Patients in the study clearly experienced exacerbations of asthma, evident in changes in symptoms, nocturnal awakenings, and use of short acting $\beta_{2}$ agonists. Changes in the symptoms score associated with an exacerbation and other markers of instability were equal to or even more pronounced than in other studies. ${ }^{7}$ Unfortunately, for technical reasons, PEF data were not available for the final analysis. With that exception, the outcome measurements used in this study were equivalent to other exacerbation studies and to common and routine clinical practice. ${ }^{9-10}$ The distinction between poor asthma control and asthma exacerbations has been defined. ${ }^{11}$ In this study some patients may have been deemed to have poor asthma control rather than an exacerbation but, given the consistent pattern of increased symptoms, the need for short acting $\beta$ agonists, and the fall in PEF, we are confident our patients did experience exacerbations. The study had adequate power to detect a difference of $30 \%$ in the proportion of patients experiencing a treatment failure between the two study arms. Although we anticipated a

\begin{tabular}{|c|c|c|c|}
\hline & $M D$ group $(n=52)$ & DD group $(n=46)$ & Total $(n=98)$ \\
\hline Mean (SD) age (years) & $32.7(11.9)$ & $31.6(14.6)$ & $32.2(13.2)$ \\
\hline No (\%) male & $13(25 \%)$ & $14(30 \%)$ & $27(28 \%)$ \\
\hline Mean FEV 1 & $2.8(0.6)$ & $2.9(0.8)$ & $2.8(0.7)$ \\
\hline \multicolumn{4}{|l|}{ Smoking status } \\
\hline Non-smokers & $43(83 \%)$ & $41(89 \%)$ & $84(86 \%)$ \\
\hline \multicolumn{4}{|l|}{ Asthma first diagnosed } \\
\hline$>1$ year ago & $47(90 \%)$ & $43(93 \%)$ & 90 (92\%) \\
\hline$\leqslant 1$ year ago & $5(10 \%)$ & $3(7 \%)$ & $8(8 \%)$ \\
\hline $\begin{array}{l}\text { Mean (SD) days from recent exacerbation } \\
\text { to visit } 1\end{array}$ & $136.6(74.1)$ & $123.7(80.1)$ & $130.6(76.8)$ \\
\hline Mean (SD) dose of budesonide at baseline & $630.8(297.4)$ & $639.1(255.1)$ & $634.7(277.0)$ \\
\hline
\end{tabular}


Table 2 Primary outcome (treatment failure) in patients randomised to the two study groups

\begin{tabular}{lcc}
\hline & $\begin{array}{l}\text { MD group } \\
(\mathbf{n}=52)\end{array}$ & $\begin{array}{l}\text { DD group } \\
\text { (n= 46) }\end{array}$ \\
\hline Asthma instability & $12(23 \%)$ & $6(13 \%)$ \\
Oral CS & $4(8 \%)$ & $7(15 \%)$ \\
Oral CS and instability & $4(8 \%)$ & $3(7 \%)$ \\
Oral CS, visit and instability & $1(2 \%)$ & $1(2 \%)$ \\
Oral CS and unscheduled visit & $0(0 \%)$ & $1(22 \%)$ \\
Unscheduled visit & $0(0 \%)$ & $1(2 \%)$ \\
Total & $21(40 \%)$ & $19(41 \%)$ \\
\hline
\end{tabular}

$M D=$ maintenance dose group; $D D=$ double dose group; $\mathrm{CS}=$ corticosteroid.

higher overall rate of exacerbations than was reported, in the end the total number of exacerbations $(n=98)$ exceeded our planned number $(\mathrm{n}=80)$.

In a smaller paediatric study of similar design, Garrett et al also found no benefit to doubling the dose of ICS at the time of an exacerbation. ${ }^{12}$ However, the authors cautioned that, because of their sample size, they did not have adequate power to detect a significant difference.

On the other hand, there is some evidence for the potential benefit of a more substantial increase in the dose of ICS such as tripling or quadrupling the maintenance dose. Foresi et $\mathrm{l}^{13}$ studied patients stabilised on $800 \mu \mathrm{g}$ budesonide twice daily who were then randomised to receive $100 \mu \mathrm{g}$ or $400 \mu \mathrm{g}$ budesonide twice a day plus additional treatment in case of exacerbation (group 1, $400 \mu$ g twice daily + placebo; group 2 , $100 \mu \mathrm{g}$ twice daily $+200 \mu \mathrm{g}$ four times daily; group 3, $100 \mu \mathrm{g}$ twice daily + placebo). Patients in group 2 who had a quadrupling of their ICS at the onset of an exacerbation had significantly better outcomes. The study by Foresi et al is not strictly comparable with ours because patients were stabilised on a high dose of budesonide before randomisation, which is not usual clinical practice. In a further study of mild exacerbations, 19 patients were randomised to either doubling the dose of ICS or addition of a single dose of $3200 \mu \mathrm{g}$ budesonide. ${ }^{14}$ Those receiving the high single dose treatment initially improved more, with a greater increase in PEF in the first week (87.4 (4.7) $\mathrm{l} / \mathrm{min} v 76.7$ (5.3) $\mathrm{l} / \mathrm{min}$, $\mathrm{p}=0.029$ ), but at 3 weeks there was no difference between the groups. A priori, we anticipated that there might be an effect on outcome based on the baseline dose of ICS. This, in fact, was true (table 3 ), with treatment failure being higher in subjects who were in the higher dose range at baseline. As might be expected, the results of this subgroup analysis were not statistically significant.

Treatment with high dose ICS has been shown to be equivalent in some studies to the administration of systemic steroids..$^{15-18}$ In one study patients discharged from the emergency department on budesonide $600 \mu \mathrm{g}$ four times daily had comparable relapse rates to patients given prednisone $40 \mathrm{mg}$ orally daily. ${ }^{15}$ In a similar study involving patients with milder asthma exacerbations, Levy et al ${ }^{16}$ showed that fluticasone $1000 \mu \mathrm{g}$ was equivalent to prednisone $40 \mathrm{mg}$ orally daily. A further paediatric study confirmed equivalence between high dose budesonide and oral prednisone. ${ }^{18}$ A synergistic effect of inhaled and oral corticosteroid was demonstrated by Rowe et a ${ }^{19}$ in acute asthma. In this study of patients discharged from the emergency department, those receiving a combination of budesonide $1600 \mu \mathrm{g}$ daily with prednisone $40 \mathrm{mg}$ daily had fewer relapses than patients receiving oral corticosteroids alone (12.8\% v 24.5\%, $\mathrm{p}=0.049)$. An early effect of ICS was demonstrated by Gibson et al in patients withdrawn from ICS and then randomised to a single dose of budesonide $2400 \mu \mathrm{g}$ or placebo. ${ }^{20}$ As early as 6 hours later there was less sputum
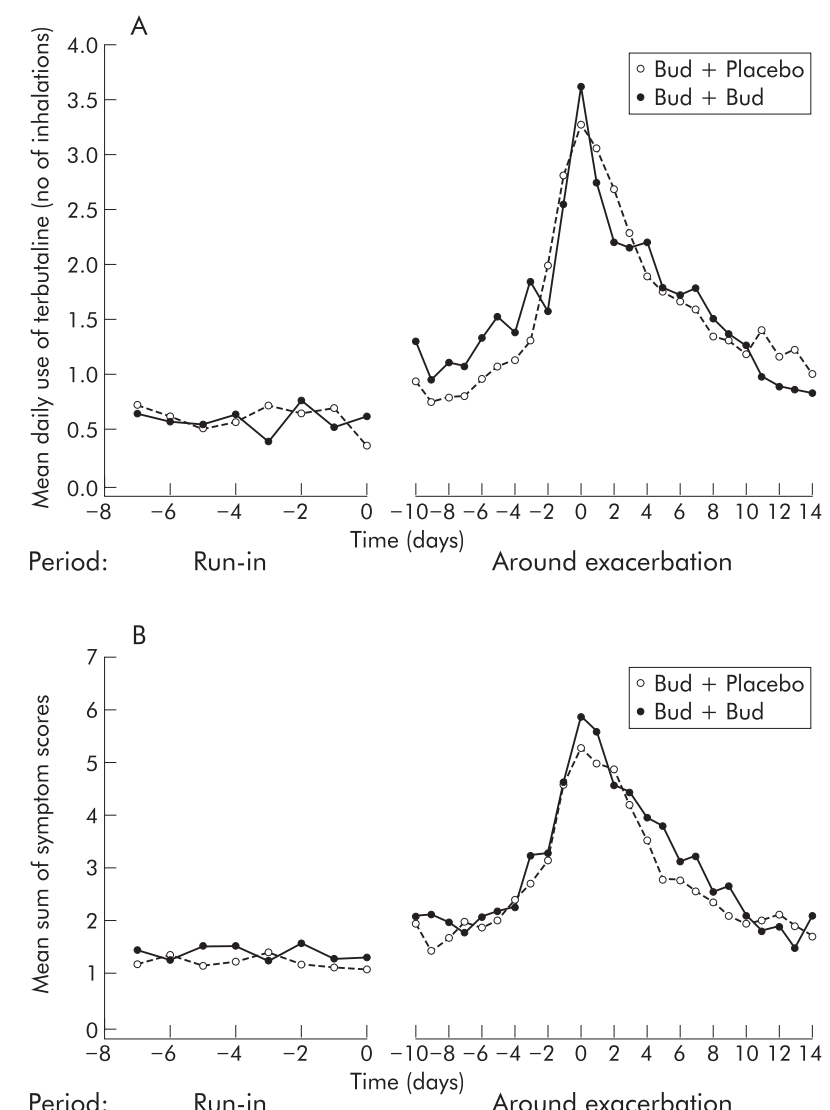

Period: Run-in Time (days) Around exacerbation

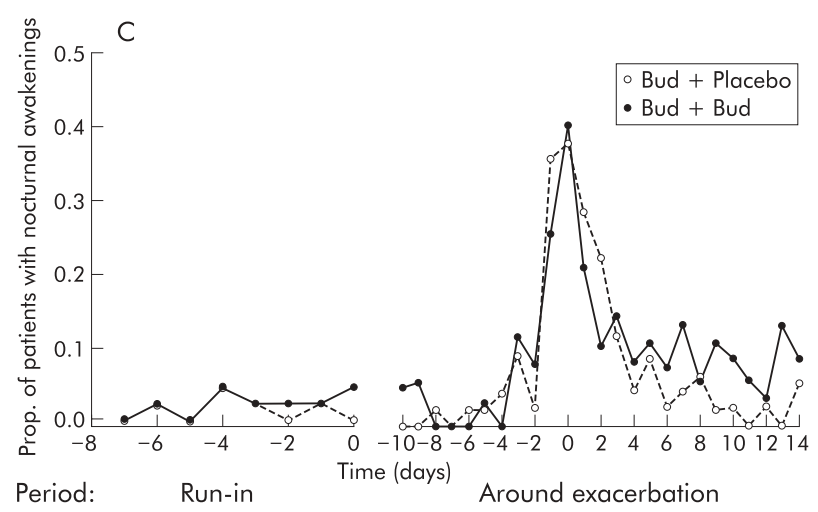

Figure 2 Pattern of $(A)$ short acting $\beta_{2}$ agonist use, (B) symptom scores, and $(C)$ nocturnal awakenings around the time of the exacerbation. Bud = budesonide.

eosinophilia and reduced airway hyperresponsiveness. All of these data suggest that higher doses of ICS may be more effective in the management of exacerbations than simply doubling the maintenance dose.

There are a number of other reasons why doubling the dose of ICS may have been ineffective in our study. Firstly, there was close monitoring and excellent compliance of the patients, although reported compliance may have overestimated actual compliance. In an effectiveness study of an action plan in a family practice setting where patients were less closely monitored, less than $40 \%$ implemented their action plan. ${ }^{21}$ On the other hand, in this study patients were alerted electronically by the MiniDoc and initiated physician contact within 48 hours of the commencement of an exacerbation. This excellent compliance in making contact 
Table 3 Treatment failure associated with initial ICS dose

\begin{tabular}{|c|c|c|c|c|c|c|c|c|c|}
\hline \multirow[b]{2}{*}{ ICS dose } & \multicolumn{3}{|c|}{ MD group $(n=52)$} & \multicolumn{3}{|c|}{ DD group $(n=46)$} & \multicolumn{3}{|c|}{ Total $(n=98)$} \\
\hline & No & Yes & $\begin{array}{l}\text { Treatment } \\
\text { failure (\%) }\end{array}$ & No & Yes & $\begin{array}{l}\text { Treatment } \\
\text { failure (\%) }\end{array}$ & No & Yes & $\begin{array}{l}\text { Treatment } \\
\text { failure }(\%)\end{array}$ \\
\hline$\leqslant 400 \mu \mathrm{g}$ & 18 & 7 & 28 & 12 & 5 & 29 & 30 & 12 & 28 \\
\hline$>400 \mu \mathrm{g}$ & 13 & 14 & 52 & 15 & 14 & 48 & 28 & 28 & 50 \\
\hline
\end{tabular}

with their physician was also reflected in their high compliance in taking maintenance ICS. The fact that the patients in the maintenance arm of the study actually took their prescribed doses of ICS may have had a significant protective effect. In a previous study of hospitalised patients, those with near fatal asthma took only $50 \%$ of their prescribed ICS, as did the control group with less severe asthma. ${ }^{22}$

Secondly, doubling the dose was associated with only twice daily administration. Previous studies have shown that a four times a day dosing regime provides greater efficacy in an acute situation or with severely uncontrolled asthma. ${ }^{23}$ However, our study was designed to assess the validity of the usual clinical recommendation, which is doubling of the daily maintenance dose and continuing the twice daily regime.

A third potential confounding factor is that patients increased their ICS up to 48 hours after the onset of the exacerbation. In clinical practice patients may increase their ICS earlier, particularly within the first 12-24 hours after the onset of increased symptoms. In some studies of exacerbations increased symptoms precede a fall in $\mathrm{PEF}^{10}{ }^{10}$ while in others the changes occur concurrently. ${ }^{24}$ It is unlikely that access to study personnel was a barrier to changes in treatment as research nurses and investigators were available on a 24 hour basis throughout the study.

There are methodological reasons which may explain our negative results. In our initial pre-study estimates, based on an earlier study, ${ }^{9}$ we estimated that $50 \%$ of patients in the MD arm would require oral corticosteroids for an exacerbation. In fact, only $17 \%$ of patients required oral steroids. This again suggests that, in an efficacy study with higher compliance with medication, regular treatment may be the most critical factor in limiting the need for augmented therapy. Although the overall result was that doubling the dose of ICS did not reduce the number of patients requiring oral corticosteroid compared with the MD group, the number of patients with unstable asthma at 14 days in the MD group $(23 \%)$ was almost double that in the DD group (13\%). An additional reason could be that only certain exacerbations respond to an increased dose of ICS. It is well documented that viral infections are responsible for many asthma exacerbations $^{25}$ and that ICS may not be effective in this type of exacerbation. ${ }^{26}$

The implications of our study are that, if patients routinely comply with maintenance ICS, then doubling the dose of ICS may not alter the course of an asthma exacerbation. Our study also shows that such patients have relatively short lived exacerbations which do not require a major change in treatment. We cannot comment on the use of larger doses of ICS for patients who do not regularly use their preventer or controller medication, or if better results might have been achieved if the dose of ICS was increased earlier. For patients experiencing an exacerbation, the clinician may choose one of several approaches including: continued close observation of the exacerbation, an empirical decision to increase the dose of maintenance ICS to doses recently studied in more severe exacerbations (for example, $\geqslant 2000 \mu \mathrm{g}$ beclomethasone equivalent), or the use of a short course of prednisone. Future prospective studies using the same methodology as outlined in this study should address the issue of the optimal increase in the dose of ICS that may be needed to prevent the development of an exacerbation or progression to a more severe exacerbation needing oral corticosteroid. A recent publication also confirms the lack of effect of doubling the dose of inhaled corticosteroids. ${ }^{27}$

\section{ACKNOWLEDGEMENTS}

The authors thank Mrs Jennifer Haddon and Dr Robert Jenkins for assistance in reviewing and preparing the manuscript, and Sergio Escobedo for assisting in the statistical analysis.

\section{Authors' affiliations}

J M FitzGerald, Respiratory Medicine, Vancouver General Hospital, University of British Columbia, Vancouver, Canada

A Becker, Section of Allergy and Clinical Immunology, Department of Pediatrics and Child Health, University of Manitoba, Winnipeg, Canada M R Sears, Department of Medicine, McMaster University and Firestone Institute for Respiratory Health, St Joseph's Healthcare, Hamilton, Canada

S Mink, Section of Respiratory Medicine, GF221 Health Sciences Centre, Winnipeg, Canada

K Chung, J Lee, AstraZeneca Canada Inc, Mississauga, Canada

AstraZeneca Canada Inc financially supported this research.

Participating investigators and sites in addition to listed authors: L-P Boulet, Ste-Foy (Québec), J-L Malo, Montréal (Québec), S Blackie, New Westminster, BC; R Olivenstein, Montréal (Québec), P-P Ernst, Montréal (Québec), K Chapman, Toronto, ON; A Mclvor, Toronto, ON; S Spier, Calgary, $A B ; M$ Yeung, Vancouver, BC.

J M FitzGerald, A Becker, M Yeung, R Olivenstein, and P-P Ernst were on the study steering committee and participated in the design of the trial. J M FitzGerald, A Becker, S Mink, M Sears, K Chung, and J Lee were involved with drafting the protocol and manuscript.

\section{REFERENCES}

1 Sears M. Natural history and epidemiology. In: FitzGerald JM, Ernst $P$, Boulet L-P, O'Byrne PM, eds. Evidence based asthma management. Hamilton: BC Decker Inc, 2000:1-12.

2 Boulet LP, Becker A, Berube D, et al. Canadian asthma consensus report 1999. Canadian Asthma Consensus Group. Can Med Assoc J 1999;161:S1-61.

3 Ernst P, FitzGerald JM, Spier S, eds. Canadian asthma consensus conference. Summary of recommendations. Can Respir J 1996;3:89-100.

4 National Heart, Lung, and Blood Institute. Global strategy for asthma management and prevention. Publication no 95-3659. Bethesda, MD: National Institutes of Health, 1995.

5 Global Initiative for Asthma (GINA). http://www.ginasthma.com.

6 BTS/SIGN. British guidelines on the management of asthma. Thorax 2003;58(Suppl I):i1-94

7 Lahdensuo A, Haahtela T, Herrala J, et al. Randomised comparison of guided self management and traditional treatment of asthma over one year. BMJ 1996;312:748-52.

8 O'Byrne PM, Barnes PJ, Rodriguez-Roisin R, et al. Low dose inhaled budesonide and formoterol in mild persistent asthma: the OPTIMA randomized trial. Am J Respir Crit Care Med 2001;164:1392-7.

9 FitzGerald J, Lee J, Becker A, et al. A randomized controlled, multicentre study to compare double dose versus maintenance of dose of inhaled corticosteroids (ICS) during asthma exacerbations. Am J Respir Crit Care Med 2000;(Suppl):A52.

10 Chan-Yeung $M$, Chang JH, Manfreda J, et al. Changes in peak flow, symptom score, and the use of medications during acute exacerbations of asthma. Am J Respir Crit Care Med 1996;154:889-93.

11 Reddel H, Ware S, Marks G, et al. Differences between asthma exacerbations and poor asthma control. Lancet 1999;353:364-9. 
12 Garrett J, Williams S, Wong C, et al. Treatment of acute asthmatic exacerbations with an increased dose of inhaled steroids. Arch Dis Child 1998;79:12-7.

13 Foresi A, Morelli MC, Catena E, on behalf of the Italian Study group. Lowdose budesonide with the addition of an increased dose during exacerbation is effective in long term asthma control. Chest 2000;117:440-6.

14 Leuppi JD, Downie SR, Salome CM, et al. A single dose of inhaled corticosteroids: a possible treatment of asthma exacerbations. Swiss Med Wkly 2002; 12:132:7-11

15 FitzGerald JM, Shragge D, Haddon J, et al. A randomized controlled trial of high dose inhaled budesonide versus oral prednisone in patients discharged from the emergency department following an acute asthma exacerbation. Can Respir J 2000;7:61-7.

16 Levy ML, Stevenson C, Maslen T. Comparison of short courses of oral prednisone and fluticasone propionate in the treatment of adults with acute exacerbations of asthma in primary care. Thorax 1996:51:1087-92.

17 Sung L, Osmond MH, Klassen TP. Randomized controlled trial of inhaled budesonide as an adjunct to oral prednisone in acute asthma. Acad Emerg Med 1998;5:209-13.

18 Nuhoglu $Y$, Bahceciler NN, Barlan IB, et al. The effectiveness of high dose inhaled budesonide therapy in the treatment of acute asthma exacerbations in children. Ann Allergy Asthma Immunol 2001;86:318-22.

19 Rowe BH, Bota GW, Fabris L, et al. Inhaled budesonide in addition to oral corticosteroids to prevent asthma relapse following discharge from the emergency department: a randomized controlled trial. J Am Med Assoc 1999;281:2119-26.

20 Gibson PG, Saltos N, Fakes K. Acute anti inflammatory effects of inhaled budesonide in asthma: a randomized controlled trial. Am J Respir Crit Care Med 2001; 163:32-6.

21 Turner MO, Taylor D, Bennett R, et al. A randomized trial comparing peak expiratory flow and symptom self-management plans for patients with asthma attending a primary care clinic. Am J Respir Crit Care Med 1998;157:540-6.

22 Turner MO, Noertiojo K, Vedal S, et al. Risk factors for near fatal asthma. A case-control study in hospitalized patients with asthma. Am J Respir Crit Care Med 1998;157:1804-9.

23 Toogood JH, Baskerville JC, Jennings B, et al. Influence of dosing frequency and schedule on the response of chronic asthmatics to aerosol steroid, budesonide. J Allergy Clin Immunol 1982;70:288-98.

24 Tattersfield AE, Postma DS, Barnes PJ, et al. Exacerbations of asthma: a descriptive study of 425 severe exacerbations. The FACET International Study Group. Am J Respir Crit Care Med 1999;160:594-9.

25 Johnston SL, Pattemore PK, Sanderson G, et al. Community study of role of viral infections in exacerbations of asthma in 9-11 year old children. BMJ 1995:310:1225-9.

26 Grunberg K, Sharon RF, Sont JK, et al. Rhinovirus-induced airway inflammation in asthma. Am J Respir Crit Care Med 2001;164:1816-22.

27 Harrison TW, Oborne J, Newton S, et al. Doubling the dose of inhaled corticosteroid to prevent asthma exacerbations: randomised controlled trial. Lancet 2004;363:271-5.

\section{LUNG ALERT}

Digital cameras are reliable in teleradiology

$\Delta$ Szot A, Jacobson FL, Munn S, et al. Diagnostic accuracy of chest X-rays acquired using a digital camera for low-cost teleradiology. Int J Med Informatics 2004;73:65-73

elemedicine, and particularly teleradiology, can be very useful in developing countries where skilled radiological expertise is not freely available. "Store and forward teleradiology", where digitised images are electronically sent after image compression to reduce file sizes (for easier internet transfer), will be particularly useful. In this article the authors assess the adequacy of such a system using a freely available digital camera and various image compression algorithms.

Ninety one erect chest radiographs, most of which had one or more features of tuberculosis (consolidation, cavitation, effusion, pneumothorax, lymphadenopathy, calcification, scarring, etc), were photographed using a 5 megapixel Olympus $3000 \mathrm{Z}$ digital camera and were converted to gray scale and appropriately modified using Adobe Photoshop software. Twenty two radiographs were normal. Four blinded independent observers (three radiologists and one pulmunologist) read three versions of the same image: (1) the original analogue image, (2) the standard JPEG image (compressed to $400 \mathrm{MB}$ ), and (3) a JPEG 2000 image which involves 60:1 wavelet image compression (compressed to $120 \mathrm{MB}$ ). Software developed by the authors (available from http://www.sourceforge.net/projects/ telemedmail) helped in image viewing.

Receiver operating characteristic curve analysis of the data showed no significant difference between the interpretation of features of tuberculosis in the three groups apart from calcification which was better detected on the standard JPEG images than on the analogue images. This study shows that low cost, small file size teleradiology allows readings of sufficient quality to make a diagnosis of tuberculosis and can be of benefit to physicians in developing countries with slow dial-up internet connections.

S Roychoudhury Specialist Registrar in Radiology, Guy's and St Thomas' Hospital NHS Trust, London, UK; shuvro@supanet.com 